\title{
Neue Waffe gegen Clostridium difficile
}

\section{Das therapeutische Armamentarium gegen den Problemkeim Clostridium difficile besteht seit kurzem nicht mehr nur aus Metronidazol und Van- comycin.}

- Infektionen mit C. difficile treten meistens bei Patienten mit lang dauernder Breitspektrum-Antibiotikatherapie auf. Grundsätzlich kann aber jedes Antibiotikum, das die Darmflora beeinträchtigt, das Risiko für diese Infektion erhöhen. Der Verlauf ist zwar in der Regel leicht und selbst limitiert, doch kann die Erkrankung auch zum Ileus, toxischen Megakolon und manchmal sogar zum Tod führen.

Fidaxomicin ist ein Antibiotikum aus der Gruppe der Makrolide, das in vitro gegen Isolate von $\mathrm{C}$. difficile achtmal potenter ist als Vancomycin. Es ist lokal im Darm wirksam und wird kaum in den Organismus aufgenommen. Fidaxomicin wurde in den USA und in der EU im Jahr 2011 zugelassen.
In einer multizentrischen, doppelblind geführten und randomisierten Nicht-Unterlegenheitsstudie wurden 535 Patienten mit Diarrhö aufgrund einer akuten, Toxin-positiven Infektion mit C. difficile entweder mit $2 \times 200 \mathrm{mg}$ Fidaxomicin oder $4 \times 125 \mathrm{mg}$ Vancomycin über zehn Tage hinweg behandelt. 509 Patienten konnten in eine ITT-Analyse einbezogen werden. Von 252 Patienten unter Fidaxomicin wiesen 221 $(87,7 \%)$ eine klinische Heilung auf im Vergleich zu 223 von 257 mit Vancomycin behandelten Patienten (86,8\%). Bezüglich der Wirksamkeit darf man also annehmen, dass Fidaxomicin mindestens so wirksam ist wie Vancomycin.

Der entscheidende Unterschied besteht allerdings in der Rezidivrate. Vier Wochen nach Beendigung der Therapie kam es bei $25,3 \%$ der Vancomycin-Patienten, jedoch nur bei 15,4\% der Fidaxomicin-Patienten zu einem klinisch manifesten Rezidiv der Clostridien-Diarrhö. 6-7\% der Patienten in beiden
Gruppen erlebten Nebenwirkungen der Therapie wie Übelkeit, Erbrechen und Bauchschmerzen.

\section{Kommentar}

Der Unterschied in der Effizienz von Fidaxomicin im Vergleich zu Vancomycin mag nach den Studiendaten gering sein; im Einzelfall kann die C.-difficile-Diarrhö aber vor allem bei alten Menschen, die mit Antibiotika behandelt wurden, ein derart gravierendes und langwieriges Problem mit weit reichenden Folgen für die Dauer des stationären Aufenthaltes und die weitere Versorgung im Pflegeheim darstellen, dass man für jede Bereicherung der therapeutischen Möglichkeiten dankbar ist.

H. S. FüESSL -
- O. A. Cornely et al.

(Korres.: oliver.cornely@ctuc.de): Fidaxomicin versus vancomycin for infection with clostridium difficile in Europe, Canada, and the USA: a double-blind, non-inferiority, randomised controlled trial. Lancet Infect Dis 2012; 12: 281-289

\section{Necrobiosis lipoidica diabeticorum}

Eine 37-jährige übergewichtige Frau mit schlecht eingestelltem Diabetes mellitus Typ 1 stellte sich mit Schmerzen im rechten Unterschenkel vor. Über der Schienbeinkante zeigte sich eine $18 \times 8$ $\mathrm{cm}$ große narbige Hautveränderung mit zentraler Atrophie und Ulzerationen. Die Plaque war bereits seit über 17 Jahren vorhanden und wurde erst in den vorausgegangenen vier Wochen schmerzhaft, nachdem es zusätzlich zu Ulzerationen gekommen war.

Eine Hautbiopsie ergab den typischen Befund einer ulzerierten Necrobiosis lipoidica diabeticorum mit teils haufenartig, teils palisadenartig angeordneter lymphatischer Zellinfiltration der Dermis. Es handelt sich um eine chronische granulomatöse Dermatitis unbekannter Ursache, die sehr häufig mit einem Diabetes mellitus assoziiert ist.

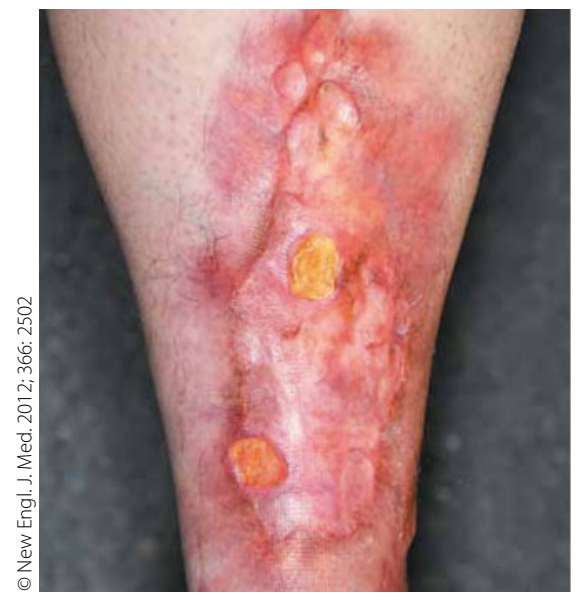

Hautveränderungen seit 17 Jahren.

Bei etwa einem Viertel der betroffenen Patienten entwickeln sich die Hautläsionen allerdings vor dem Auftreten des Diabetes. Sie sind gelbbraun verfärbt, weisen Teleangiektasien, eine zentrale Atrophie und erhabene, livide verfärbte Ränder auf. Die häufigste Lokalisation ist über dem Schienbein und am Fußrücken. Bei etwa einem Drittel der Patienten entstehen Ulzerationen, die meistens durch ein Trauma ausgelöst werden. In seltenen Fällen kann sich auch ein Plattenepithelkarzinom der Haut entwickeln, vor allem auf dem Boden lange bestehender ulzerierter Läsionen. Mittels einer feuchten Wundbehandlung und Abtragung der Nekrosen erzielte man bei der Patientin eine Schmerzerleichterung. Der weitere Verlauf konnte nicht verfolgt werden, da die Patientin sich nicht mehr vorstellte.

\section{H. S. FÜESSL .}

- J. Dissemond

(University of Essen, Essen, germany): Necrobiosis lipoidica diabeticorum. New Engl. J. Med. 2012; 366: 2502 